\title{
Central Controller Framework for Mobile Cloud Computing
}

\author{
Debabrata Sarddar ${ }^{1}$, Priyajit $\operatorname{Sen}^{2}$ and Manas Kumar Sanyal ${ }^{3}$ \\ ${ }^{* 1}$ Assistant professor, Department of Computer Science and Engineering, \\ University of Kalyani \\ ${ }^{* 2}$ Student of Master of Technology, Department of Computer Science and \\ Engineering, University of Kalyani \\ ${ }^{* 3}$ Professor, Head and Dean (Eng-Mgmt-Tech, KU), Department of Business \\ Administration, University of Kalyani
}

\begin{abstract}
Now-a-days Mobile-Cloud Computing is a global computing infrastructure. MobileCloud computing is the combination of mobile computing and cloud computing. Today's smart phones are facing problem of small size and low battery life. One of the solutions to the problem of size of the mobile devices is by using the mobile device via extra touchpad device either by wireless fidelity network or Bluetooth. Low battery can be replaced by a powerful battery. The problem in cloud network is the higher response time of nodes while performing data communication through co-operative caching. This problem can be overcome by placing a Central Controller device as a hub in star topology network. The Central Controller device keeps track of the status of all other devices during the communication by running passive scan over the devices.
\end{abstract}

Keywords: Mobile Cloud Computing, Co-operative Cache, Star Topology Network, Response time, Wireless Fidelity, Bluetooth, Central Controller

\section{Introduction}

Mobile cloud computing (MCC) is an infrastructure where both the data storage and data processing happen outside of the mobile device. Mobile cloud applications move the computing power and data storage away from the mobile devices into powerful and centralized computing platforms located in clouds, which are then accessed over the wireless connection based on a thin native client. Mobile devices face many resource challenges (battery life, storage, size etc.) [1]

According to a recent study by ABI Research, more than 240 million businesses will use cloud services through mobile devices by 2015.That traction will push the revenue of mobile cloud computing to $\$ 5.2$ billion. It is necessary to have a secured and balanced access over the information to remain a business effective, competitive and profitable. Cloud computing provides a solution to minimize the cost of accessing resources and maximize the resource utilization. New software tools are available from remote locations so deploying a costly resource is not needed [16]. Many security issues are there as different technologies including networks, databases, operating systems, virtualization, resource scheduling, transaction management, load balancing, concurrency control and memory management are used in cloud computing. Some of the security issues include data isolation, browser security, malware, flooding attack, privacy, data integrity, and protection of DATA [15]. 
i) Problem for Low Battery Life of Mobile Devices:

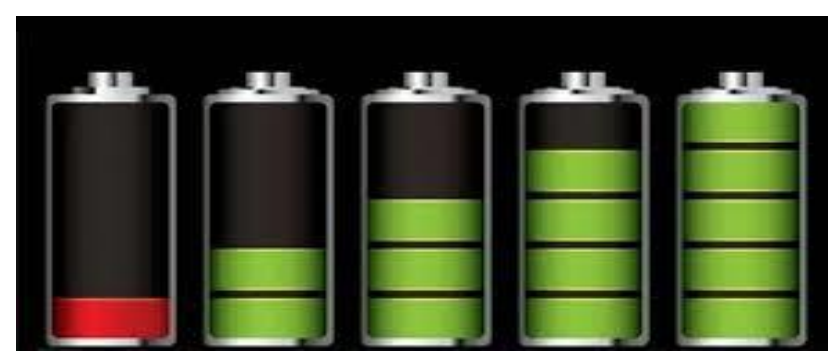

Figure 1. Low Battery Problem

ii) Problem for Small Size of Mobile Devices:

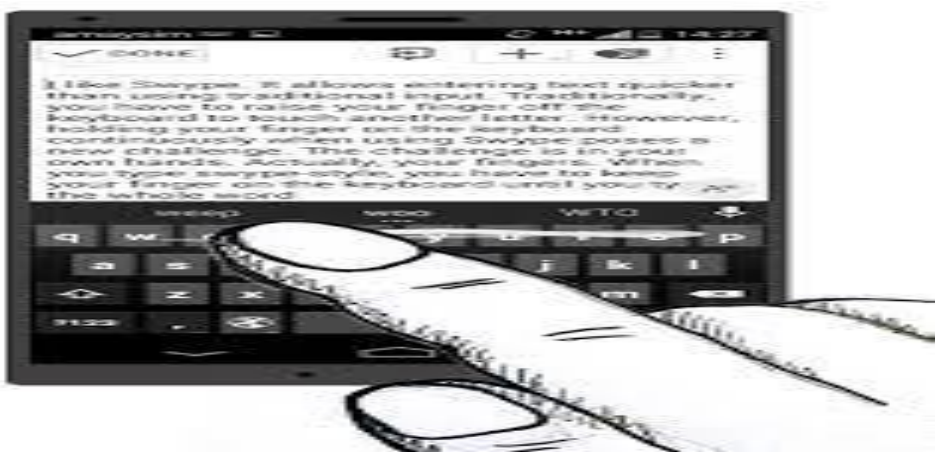

Figure 2. Small Size Problem

\section{iii) Mobile Cloud Computing Architecture:}

In Mobile Cloud Computing (MCC) architecture Mobile devices are connected to the mobile networks via base stations that establish and control the connections and functional interfaces between the networks and mobile devices.

Mobile users' requests and information are transmitted to the central processors that are connected to servers providing mobile network services. The subscribers' requests are delivered to a cloud through the Internet. In the cloud, cloud controllers process the requests to provide mobile users with the corresponding cloud services [1].

\section{Architecture}

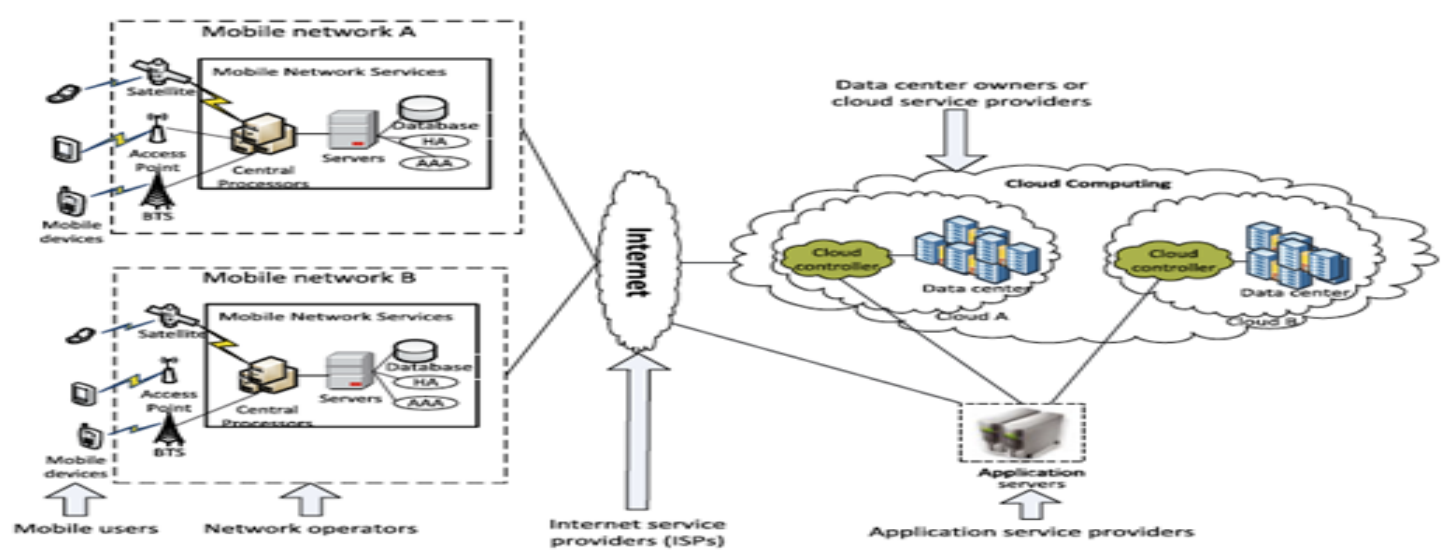

Figure 3. MCC Architecture 


\section{iv) Star Topology Network:}

In a star topology, each device has a dedicated point to point link only to a central controller, usually called a hub. The devices are not directly linked to each other.

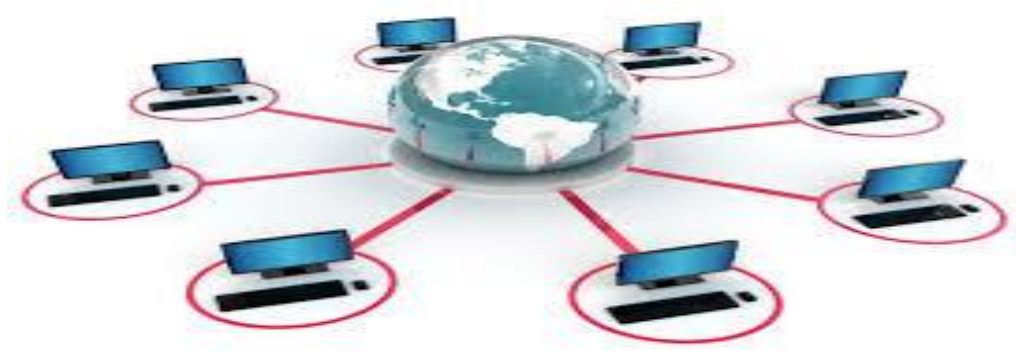

Figure 4. Star Topology Network

\section{v) Cloud Service Hierarchy:}

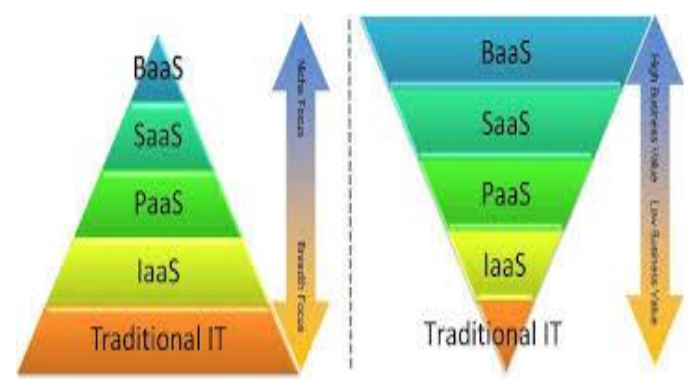

Figure 5. Cloud Service Hierarchy

a) IAAS: IAAS provides storage, hardware, servers and networking components to the user. For example elastic cloud of Amazon.

b) PAAS: PAAS provides an environment of parallel programming design, testing etc. For example Google App engine.

c) SAAS: SAAS provides some applications that can be used via Internet and is paid according to the usage. For example Google Online Office [15].

vi) Deployment Models:

a) Public Cloud: A type of cloud in which the cloud services are delivered over a network which is open for public usage.

b) Private Cloud: A type of cloud that is implemented on a cloud-based secure environment that is safeguarded by a firewall. Private cloud as it permits only the authorized users can use the data.

c) Community Cloud: A type of cloud in which the setup is mutually shared between many organizations that belong to a particular community.

d) Hybrid Cloud: A type of cloud which is integrated. It can be an arrangement of two or more cloud servers, i.e. private, public or community cloud that is bound together but remain individual entities. 


\section{vii) WLAN Scanning Process:}

a) Active Scan: In Active Scanning a station finds out network rather than waiting for network to announce its availability to all the stations.

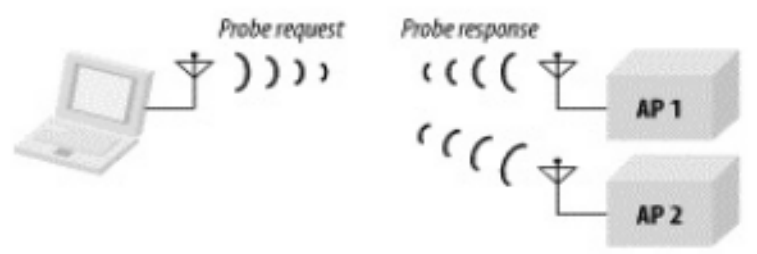

Figure 6. Active Scanning Process

b) Passive Scan: In Passive Scanning, WLAN station moves to each channel as per channel list and waits for beacon frames. All these frames are buffered and are used to decode and extract information about base stations.

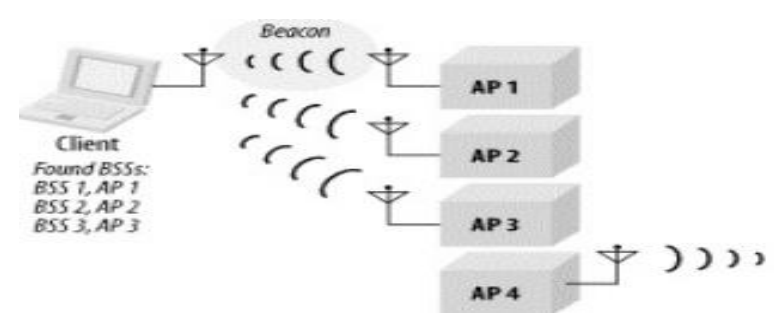

\section{Figure 7. Passive Scanning Process}

The rest of the paper is organized as follows, Section II overviews on the related work. Section III is the proposed work. Section IV is the discussion about the work. Section V is the conclusion. Section VI focuses on the future work to be carried out. Section VII contains references and Section VIII contains Author's profile.

\section{Related Work:}

Co-operative caching improves the response time by reducing VM synthesis time by caching the previous state [1]. Co-operative caching consists of multiple distributive caches to improve system response time [1]. In cloudlet, when a mobile client requests for a cloud service, the network searches for the data in the local cloud. If the service is not available, the users should contact a distinct cloud that involves network transfer and latency. The authors of research paper [17] gives a survey of MCC, which helps general readers, have an overview of the MCC including the definition, architecture, and applications. In paper [18] the authors have discussed about the architecture of mobile cloud computing (MCC) with the different services needed for the client and server in MCC. Some of the research works were done over billboard manager which is used to handle a large number of storage nodes. Billboard manager knows the available blank space of cloud storage [15].

\section{Proposed Approach:}

The Limitations of today's smart phones are mainly of smaller size and low battery life. While typing on mobile devices, it often creates problem due to small size of typing pad in mobile device. To overcome the problem of the size, the mobile phone can be used via touchpad device connected through WIFI hotspot. A touchpad device can be used, where processor and storage both will be in the mobile device but virtually we can use the 
touchpad while typing in mobile devices. The touchpad device can be useful while travelling in vehicles. Even many more touchpad devices can be used to get the benefit of parallel computing in a single mobile device.

Second problem is low battery life of mobile devices. To overcome this problem low battery can be replaced by a powerful battery as required for the mobile device so that it can provide backup for at least 3-4 day. In this case cost will increase but the performance, battery backup, user attractiveness will also increase.

In cloud computing, instead of co-operative caching one of the devices can be chosen as a central device called 'Central Controller' that keeps track of the status of all the other devices connected with it using a star topology network. During this time the Central Controller scan passively all the devices dynamically to get the current status about storage whereas a device need to store any data will perform an active scan over the Central Controller to know the status of all other devices. As soon as, it gets the information it will proceed directly onto that particular device to store the data. Thus Response time and efficiency can both be effective and valuable for future course. The algorithm is discussed below:

\section{Algorithm:}

1. A finite set of nodes $S=\{\mathrm{N} 1, \mathrm{~N} 2, \mathrm{~N} 3, \ldots \ldots \ldots .$.

2. Central Controller $(\mathbf{N})$ is connected with all the nodes present in set $\mathbf{S}$ using star topology network.

3. Central Controller (N) runs a passive scan over all other devices dynamically to achieve information about the status all the devices connected with it and always keep updated information.

4. A node $\mathrm{N}_{\mathrm{i}}\left(\mathrm{i}=1,2,3,4,5, \ldots \ldots \ldots\right.$ ). want to store the data to any other device $\mathrm{N}_{\mathrm{j}}$ $(\mathrm{j}=1,2,3,4,5, \ldots \ldots . . . w h e r e \mathrm{j} \neq \mathrm{i})$ runs an active scan over Central Controller(N) to achieve the information about the status of rest other devices.

5. When the status of all the nodes were obtained, the node that want to store the data will directly choose the node having large storage space and it store the data within that node.

6. Continue step 4 and 5 until data communication over.

7. End

\section{Connection between Central Controller and Nodes in the Network:}

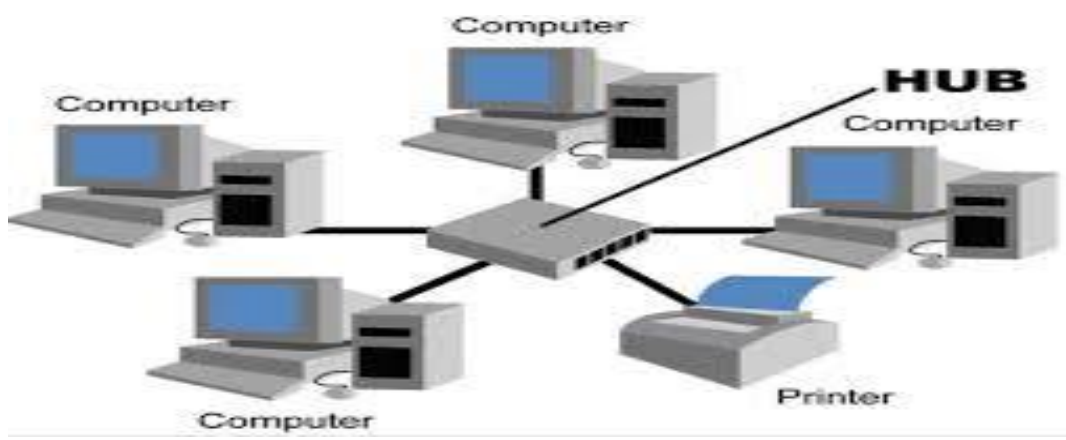

Figure 8. Connection between Central Controller and other Nodes in the Network 


\section{Flowchart:}

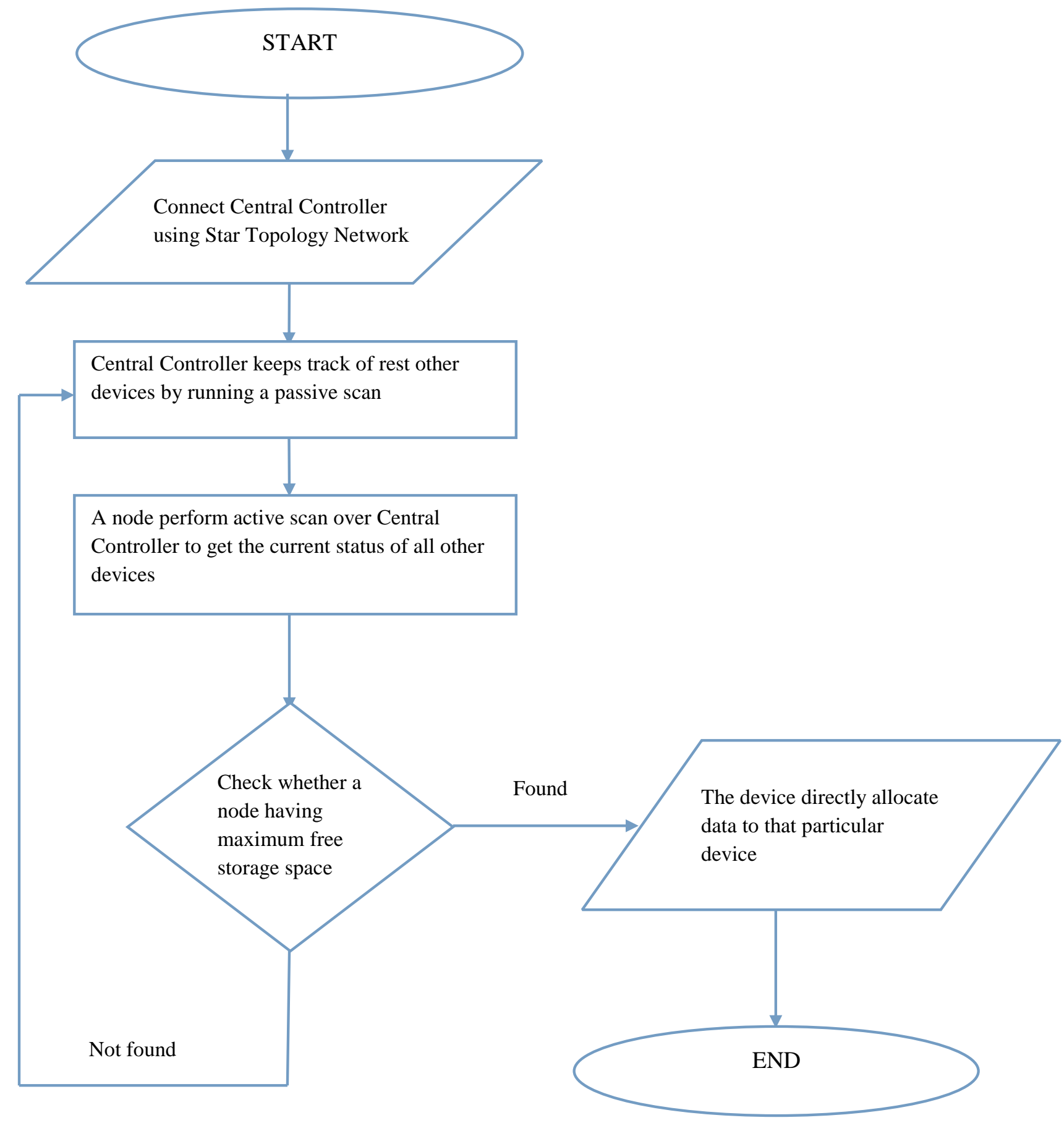

Figure 9. Flowchart of Central Controller Concept

\section{Discussion:}

The main drawbacks of today's mobile devices can be solved. The different solutions of the problem are discussed below:

A mobile device is used through a touchpad via wireless network is a solution to the problem of small size of the mobile devices. Mobile devices are portable. A touchpad device can be used while typing a message at very high speed. Less time will be required while typing. A powerful battery can be placed in smart phones depending on the need to obtain long battery backup. Instead of co-operative caching framework as described by 
the author [1], Devices may be connected with a central device and the central device keeps updated information about all other devices connected in the network. The central controller performs a passive scan over the devices to obtain the status of the other devices. When a device need to obtain the status of the devices it performs an active scan over the Central Controller to achieve the information as discussed by the authors of paper [15]. Thus efficiency of the system can be increased. The process of data communication will be faster. Performance will increase.

\section{Conclusion}

Central Controller concept will increase the performance, efficiency, response time and it provides easy access to other devices to obtain the status of devices that are connected by a star topology network with the central controller. In this case cost may increase but the overall speed up of the system will also increase. Again by increasing the power of the battery, the battery life can also be increased so that users of the Smartphone devices do not face problem of recharging very frequent. A touchpad device can be used with smart phones via wireless network to overcome the problem of typing in the mobile device. We expect that our proposal should be taken for future prospect.

\section{Future Work:}

Future works can be carried out over the central controller concept to minimize the latency and maximize the performance of the system. There are some issues related to the Central Controller device. Those issues are: If any problem is detected in the central controller device then how to solve the problems regarding the central controller? Central controller focuses on some of the security issues. Such as storage security, middle ware security, data security, network security and application security. How to enhance the security further will be the future prospect of this paper.

\section{References}

[1] P. Theresa Joy and K. Poulose Jacob, "Cooperative Caching Framework for Mobile Cloud Computing", Global Journals Inc.(USA), Version 1.0, Online ISSN: 0975-4172 \& Print ISSN: 0975-4350, vol. 13, no. $8,(\mathbf{2 0 1 3})$.

[2] S. Shafique Qureshi, "Mobile Cloud Computing as a Future for Mobile Applications-Implementation methods and Challenging Issues", Proc. of IEEE CCIS2011.

[3] P. Bhal, "Advancing the state of mobile cloud computing", Proceedings of the Third ACM workshop on MobileCloud Computing and services(New York, NY, USA 2012), MCS '12, ACM, pp. 21-28.

[4] R. Buyya, C. S. Yeo, S. Venugopal, J. Broberg and I. Brandic, "Cloud Computing and emerging IT platforms: Vision, hype, and reality for delivering computing as the $5^{\text {th }}$ utility", Future Generation Computer Systems, vol. 25, pp. 599-616.

[5] J. Han, W. Susilo and Y. Mu, "Identity-based data storage in cloud computing", Future Generation Computer Systems, doi: 10.1016/j.future.2012.07.010, vol. 29, (2013), pp. 673-681.

[6] A. Kumar, "World of Computing and Security", International journal of Cloud Computing and Services Science, vol. 1, no. 2, (2012), pp. 53-58.

[7] P. Zhang Zhao, G. Cao and C. R. Das, "Cooperative caching in wireless P2P networks: design, implementation and evaluation”, IEEE Trans Parallel Distributed Syst., vol. 21, no. 2, (2010), pp. 229.

[8] R. Bose, D. Sarddar and S. Sahana, "Billboard Manager based tools helps the critical virtual machines to migrate in appropriate cloud nodes automatically", International Journal of Scientific Research in Computer Science Applications and Management Studies, ISSN: 2319-1953, vol. 4, no. 2, (2015) March, pp. 4-7.

[9] D. Sarddar and R. Bose, "Architecture of Server Virtualization Technique Based on VMware ESXI server in the Private Cloud for an Organization", International Journal of Innovation and Scientific Research, ISSN 2351-8014, vol. 12, no. 1, (2014) November, pp. 284-294.

[10] D. Sarddar and R. Bose, "Secure Co-processor and Billboard Manager Based Architecture Help to Protect \& Store the Citrix Xenserver Based Virtual Data", COMPUSOFT, An international Journal of advanced computer technology, ISSN Online: 2320-0790, vol. 3, no. 1, (2014) January, pp. 473-479.

[11] X. Dutreild, N. Rivierre, A. Moreau, J. Malenfant and I. Truck, "From data center resource allocation to control theory and back", Proc. IEEE 3rd Int. Conf. on Cloud Computing, (2010), pp. 410-417. 
[12] D. P. Anderson, "BOINC: A system for public-resource computing and storage", Proc. $5^{\text {th }}$ IEEE/ACM Int. Workshop on Grid Computing, (2004), pp. 4-10.

[13] D. Sarddar and E. Nandi, "Cloud Billing \& Verification of consumed resources and spaces by a cloud user", International Journal for Research and Development in Technology, Inter, ISSN:2349-3585, vol. 4, no. 4, (2015) October, pp. 67-76.

[14] D. Sarddar and E. Nandi, "Efficient Optimization Server Selection Technique in Content Delivery Network", International Journal of Mathematical sciences and computing, pp. 23tion of Edge 1-10. DOI: 10.5815/ijmsc.2015.04.01, vol. 4, no. 5, (2015) November.

[15] D. Sarddar and R. Bose, "Creating a Secured Cloud Based Data Center Using Billboard Manager (BM) and Secure Co-Processor", International Journal of Scientific \& Engineering Research, ISSN 2229-5518, vol. 4, no. 12, (2013) December.

[16] R. Bose, H. Biswas and D. Sarddar, "A new Approach in Delivering Building Information Modeling Applications and Data to Remote Users over Cloud through Billboard Manager", International Journal of Computer Applications(0975-8887), vol. 119, no. 14, (2015) June.

[17] D. Sarddar and R. Bose, "A Mobile Cloud Computing Architecture with Easy Resource Sharing", International Journal of Current Engineering and Technology, E-ISSN 2277-4106, P-ISSN 2347-5161, 2014 INPRESSCO ${ }^{\circledR}$.

[18] http://www.smartdevelopments.org.

[19] http://www.vodafone.com[17].

\section{Authors}

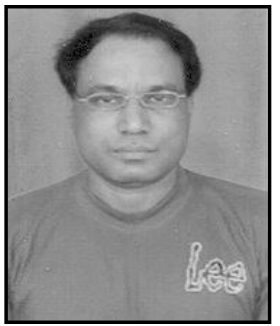

Debabrata Sarddar, Assistant Professor in the Department of Computer Science and Engineering, University of Kalyani, Kalyani, Nadia, West Bengal, India. He has done PhD from Jadavpur University. He completed his M.Tech in Computer Science \& Engineering from DAVV, Indore in 2006, and his B.E in Computer Science \& Engineering from NIT, Durgapur in 2001. He has published more than 150 research papers in different journals and conferences. His research interest includes Mobile Computing, Wireless Sensor Network and Cloud Computing.

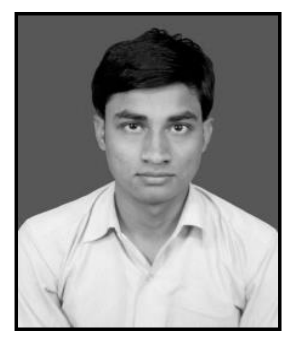

Priyajit Sen is presently pursuing M.Tech in Computer Science and Engineering at the Department of Computer Science and Engineering, University of Kalyani, Kalyani, Nadia, West Bengal, India. He completed his MCA from Department of Computer Science and Engineering, University of Kalyani, Kalyani, Nadia, West Bengal, India in 2015. His research interest includes Mobile Computing, Wireless Sensor Network and Cloud Computing.

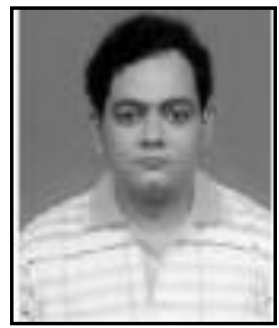

Dr. Manas Kumar Sanyal is currently working as a professor and Head of the Department in the Business Administration, Kalyani University. He is currently the Dean of faculty of Enginnering, Technology and Management of the University of Kalyani. He has completed M.Tech. in Computer Science from Calcutta University and Ph.D. He is serving more than 18 years in teaching. He has presented papers in national and international Conferences and published papers in international journal. His area of interest is e-Governance, E-Commerce, ERP, Data warehouse, cloud computing, Semi Structured Data base. 